\title{
Novel encapsulation technique for incorporation of high permittivity fillers into silicone elastomers
}

\author{
Mazurek, Piotr Stanislaw; Hvilsted, Søren; Skov, Anne Ladegaard
}

\section{Published in:}

Proceedings of SPIE

Link to article, DOI:

$10.1117 / 12.2044778$

Publication date:

2014

Document Version

Early version, also known as pre-print

Link back to DTU Orbit

Citation (APA):

Mazurek, P. S., Hvilsted, S., \& Skov, A. L. (2014). Novel encapsulation technique for incorporation of high permittivity fillers into silicone elastomers. In Proceedings of SPIE: Electroactive Polymer Actuators and Devices (EAPAD) XVI (Vol. 9056). SPIE - International Society for Optical Engineering. https://doi.org/10.1117/12.2044778

\section{General rights}

Copyright and moral rights for the publications made accessible in the public portal are retained by the authors and/or other copyright owners and it is a condition of accessing publications that users recognise and abide by the legal requirements associated with these rights.

- Users may download and print one copy of any publication from the public portal for the purpose of private study or research.

- You may not further distribute the material or use it for any profit-making activity or commercial gain

- You may freely distribute the URL identifying the publication in the public portal 


\title{
Novel encapsulation technique for incorporation of high permittivity fillers into silicone elastomers
}

\author{
Piotr Mazurek, Søren Hvilsted, Anne Ladegaard Skov* \\ Technical University of Denmark, Department of Chemical and Biochemical Engineering
}

\begin{abstract}
The research on soft elastomers with high dielectric permittivity for the use as dielectric electroactive polymers (DEAP) has grown substantially within the last decade. The approaches to enhance the dielectric permittivity can be categorized into three main classes: 1) Mixing or blending in high permittivity fillers, 2) Grafting of high permittivity molecules onto the polymer backbone in the elastomer, and 3) Encapsulation of high permittivity fillers. The approach investigated here is a new type of encapsulation which does not interfere with the mechanical properties to the same content as for the traditionally applied thermoplastic encapsulation. The properties of the elastomers are investigated as function of the filler content and type. The dielectric permittivity, dielectric loss, conductivity, storage modulus as well as viscous loss are compared to elastomers with the same amounts of high permittivity fillers blended into the elastomer, and it is found that the encapsulation provides a technique to enhance some of these properties.
\end{abstract}

Keywords: DEAP, PDMS, encapsulation, filler, relative permittivity, microfluidic device

\section{INTRODUCTION}

There is no unequivocal answer to the question of what increases actuation performance of dielectric electroactive polymers most efficiently. Scientists allover the globe discuss this issue presenting new ways of handling these materials but non of them seems to outdistance other methods. The actuation performance of DEAP, which in another words can be defined as strain produced on elastomer when applying electric field and can be defined as:

$$
S=\frac{\varepsilon_{0} \varepsilon_{r}}{Y}\left(\frac{V}{d}\right)^{2}
$$

where $\varepsilon_{0}$ and $\varepsilon_{\mathrm{r}}$ are vacuum permittivity and relative permittivity, respectively. $\mathrm{Y}$ is the Young's modulus of material, $\mathrm{V}$ is applied voltage and $\mathrm{d}$ thickness of investigated elastomer. According to Equation 1 there are four parameters that can be altered in order to increase the actuation performance. These are $\varepsilon_{\mathrm{r}}, \mathrm{Y}, \mathrm{d}$ and the maximal value of quotient $\mathrm{V} / \mathrm{d}$ which is defined by breakdown strength of polymer composition. Goswami et al. discussed merit of varying these parameters and concluded that modification of relative permittivity of material gives most prominent results and leaves most room for improvements [1]. The relative permittivity $\left(\varepsilon_{r}\right)$ is a parameter that together with vacuum permittivity $\left(\varepsilon_{0}=8.85 \times 10^{-12} \mathrm{~F} / \mathrm{m}\right)$ defines the permittivity of the material ( $\varepsilon$, Equation 2$)$. Relative permittivity is directly related to electric susceptibility $(\chi)$ which is a measure of degree of polarization (polarization density $P$, Equation 3 ) of a dielectric which finally is a property that describes actuation capability of dielectric materials when exposed to electric field $\mathrm{E}$.

$$
\begin{aligned}
& \varepsilon=\varepsilon_{0} \varepsilon_{r}=\varepsilon_{0}(1+\chi) \\
& P=\varepsilon_{0} \chi E
\end{aligned}
$$


Different approaches have been performed in order to increase relative permittivity of dielectric elastomers. One of the most frequently reported methods is based on mixing elastomers with high permittivity fillers like carbon nanotubes, carbon black, titanium dioxide etc. [2,3]. Composites containing different amounts of fillers can potentially improve not only the actuation performance but also mechanical properties of final material. Increasing attention is also attracted towards grafting of polar molecules onto the silicone polymer backbone which significantly improves the dielectric permittivity without compromising its high inherent breakdown strength and increasing dielectric loss [4]. It was also reported that grafting of less than 0.5 wt.\% of push-pull dipols onto a crosslinker of the silicones can enhance the dielectric constant by $19 \%[5,6]$.

As alternative to methods described above, Opris et al. [7] presented a novel way of incorporation of conductive fillers encapsulated within an insulating shell into elastomer networks. In their approach poly(aniline) (PANI) was encapsulated in poly(divinyl benzene) (PDVB) forming capsules of less than $1 \mu \mathrm{m}$ diameter, which were later dispersed in poly(dimethylsiloxane) (PDMS) films. In this way the filler particles were efficiently separated from each other by non-conductive layers preventing formation of conductive paths throughout the material, which may lead to significant and uncontrolled increase of dielectric loss as well as decrease of breakdown strength of materials. By applying this technique they successfully improved the relative permittivity of PDMS based composites keeping breakdown strength at an acceptable level.

In this paper we focus on preparation of liquid-core microcapsules of extremely narrow size distribution and incorporating them into PDMS matrix. We show how different amounts of encapsulated filler influence mechanical and electrical properties of the formed composites. We present an efficient method of encapsulation of high permittivity polar liquids into PDMS shells. The shells allow for further reaction since they are prepared from an elastomer with excess of silicone hydrides. These will allow for further crosslinking by covalent grafting into the matrix of silicone elastomer possessing excess of vinyl groups. The excess of vinyl groups in PDMS compositions still allows for formation of strong and elastic networks [8].

The core-shell microspheres (water surrounded by crosslinked PDMS) were prepared with the help of a flowfocusing microfluidic device, which has previously been reported as a successful tool for preparation of microcapsules of extremely narrow size distribution [9]. The composition used for preparation of a microfluidic chip presented in this report is based on thiol and allyl terminated compounds mixed in stoichiometric imbalance and then crosslinked under UV-irradiation. The preparation procedure of this rapidly curable and solvent resistant composition was presented and described by Haraldsson et al. [10]. Thiol-ene "click chemistry" was also used for surface modification of channels in the microfluidic chip.

\section{EXPERIMENTAL DETAILS}

\subsection{Materials}

Sylgard 184 silicone kit used for preparation of mirror-image mold was purchased from Dow Corning. All three monomers used for preparation of the microfluidic chip polymer matrix (pentaerythritol tetrakis(3-mercaptopropionate) (PETMP), triallyl-1,3,5-triazine-2,4,6(1H,3H,5H)-trione (TATATO) and bisphenol A diglycidyl ether (BADGE)) as well as monomers used for grafting reactions (allylmalonic acid and 2,2,3,3,4,4,5,5,6,6,7,7-dodecafluoroheptyl acrylate (DFHA)) were purchased from Sigma Aldrich. Thiol-ene reaction photoinitiator Lucirin TPO-L was obtained from BASF GmbH Germany. Grafting reaction photointiator 2,2-dimethoxy-2-phenylacetophenone (DMPA), thiol-epoxy reaction catalyst 1,5-diazabicyclo(4.3.0)non-5-ene (DBN), sodium hydroxide, sodium dodecyl sulfate (SDS), poly(vinyl alcohol) (PVA) as well as all solvents used in this study were obtained from Sigma Aldrich. Structural formulas of the three compounds forming the microfluidic chip matrix can be seen in Figure 1. 

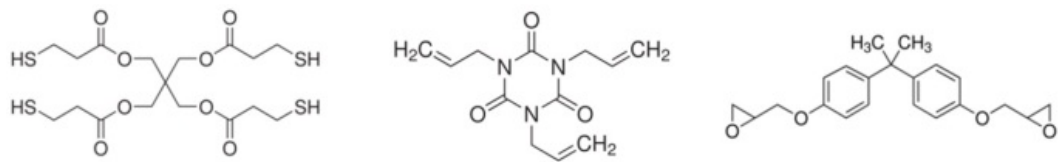

Figure 1: Structural formulas of compounds used for preparation of flow-focusing microfluidic chip (tetrathiol (left), triallyl (middle), diepoxy (right)).

\subsection{Microfluidic chip preparation}

Mold preparation. After the master-mold was designed, all channels and geometries were micro-milled in a poly(methyl methacrylate) (PMMA) plate. Sylgard 184 silicone kit was used for producing the mirror-image mold. Base and crosslinker were mixed together in $10: 1$ weight ratio respectively, degassed and casted on the PMMA master-mold. The PDMS curing reaction was allowed to proceed for two hours at $120{ }^{\circ} \mathrm{C}$.

Crosslinking reaction step. The off-stoichiometric mixture of monomers used for preparation of microfluidic chip consisted of pentaerythritol tetrakis(3-mercaptopropionate), triallyl-1,3,5-triazine-2,4,6(1H,3H,5H)-trione and bisphenol A diglycidyl ether (BADGE) (molar ratio of reactive groups was $2: 1: 0.15$, respectively). Lucirin TPO-L was used as a photoinitiator for the thiol-ene crosslinking reaction and DBN was introduced to accelerate the thiol-epoxy reaction. Schematic thiol-ene and thiol-epoxy reactions are presented in Figure 2.

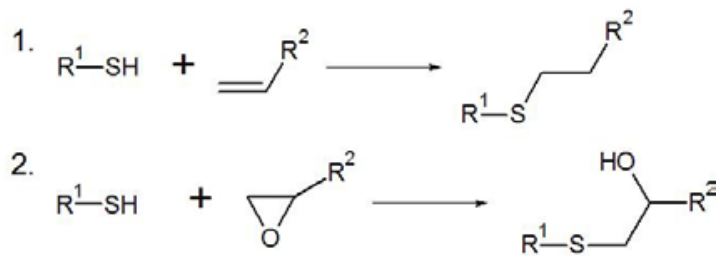

Figure 2: 1) The thiol-ene photoinitiated reaction; and 2) the DBN catalyzed thiol-epoxy reaction.

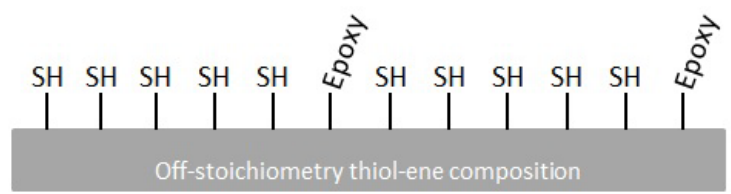

Figure 3: Schematic illustration of UV-cured network resulting from off-stoichiometric mixture of tetrathiol, triallyl and diepoxy compounds ( $2: 1: 0.15$, respectively).

The composition of all five compounds was thoroughly mixed, degassed and transferred onto the PDMS mirror-image mold and exposed to UV-irradiation for a few minutes at light intensity of $\sim 4 \mathrm{~mW} / \mathrm{cm}^{2}$ in order to initiate the crosslinking reaction between thiol and allyl components exclusively. Schematic illustration of the obtained network is presented in Figure 3. After two wafers of microfluidic chip were cured, they were assembled together (avoiding formation of air voids) and placed in the oven for 2 hours at $80{ }^{\circ} \mathrm{C}$. In this step the thiol and epoxy groups present on the surfaces of both wafers react with each other assuring not only covalent bonding of the two wafers but also increase of degree of crosslinking resulting in increased glass transition temperature and stiffness of the material. The amount of BADGE used in this system was kept at the lowest possible level leaving as many thiol groups on the surfaces of the channels as possible. The remaining thiol groups made it possible to modify the surface of the channels later, which is a crucial step in the preparation of flow-focusing microfluidic chips. 
Surface modification of microfluidic chip channels. The surface grafting reaction follows the same thiol-ene chemistry mechanism as presented above for the crosslinking reaction of the main matrix. In this system we take advantage of the thiol groups remaining on the surface of the material and induce the grafting reaction by injecting different grafting solutions into the chip channels. Structural formulas of the grafted compounds can be seen in Figure 4. In the first step of the process the $10 \mathrm{wt} . \%$ solution of DFHA in ethanol with additional $1 \mathrm{wt} . \%$ DMPA as photoinitiator was prepared. After the channels were filled with the solution, a part of the chip that was intended to remain hydrophilic was covered with a stencil mask in order to block the grafting reaction in that area. Subsequently the chip was exposed to UVirradiation at light intensity of $\sim 4 \mathrm{~mW} / \mathrm{cm}^{2}$. In the following step the channels were thoroughly washed with substantial amounts of ethanol to dispose of the grafting solution. Afterwards a $10 \mathrm{wt} . \%$ solution of allylmalonic acid in ethanol with 1 wt.\% DMPA was prepared and injected into the channels of the chip. The area of the chip that was designed to be hydrophobic was masked with stencil mask to avoid grafting reaction of allylmalonic acid in that area. The chip was again exposed to UV-irradiation and after the reaction was complete, it was flushed with ethanol. Subsequently a $0,01 \mathrm{M}$ $\mathrm{NaOH}$ aqeous solution was injected into the channels in order to replace protons of the carboxylic groups with sodium cations. This process was proved to significantly increase the hydrophilicity of the material.
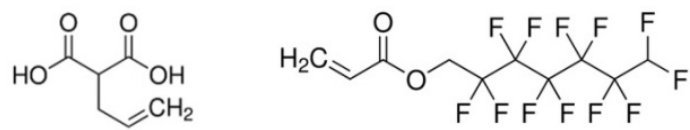

Figure 4: Structural formulas of allylmalonic acid and 2,2,3,3,4,4,5,5,6,6,7,7-dodecafluoroheptyl acrylate.

\subsection{Formation of double emulsion in microfluidic chip cross-junctions}

After microfluidic chip fabrication (see scheme of the chip in Figure 5) it was sandwiched between custom made polycarbonate holder plate equipped with three inlet holes and one outlet hole. All liquids were introduced to the chip with the help of separately controlled Harvard Apparatus 11 plus syringe pumps capable of precise adjusting flow rates.

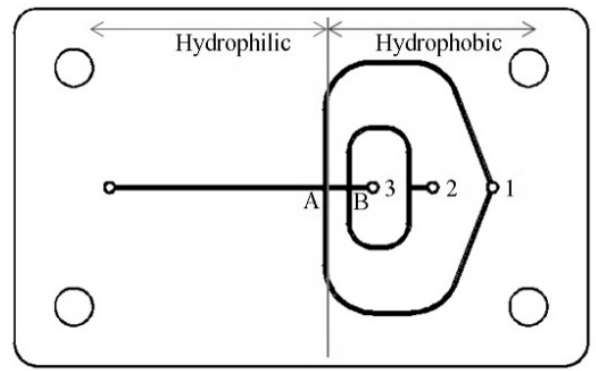

Figure 5: Schematic visualization of wetting properties of the channel surfaces in microfluidic chip used for preparation of o/w and w/o/w emulsion where $\mathrm{o}=\mathrm{oil}$ and $\mathrm{w}=$ water. Chip dimensions are 47 x $29 \mathrm{~mm}$ with $280 \mu \mathrm{m}$ broad channels.

SDS/PVA (3 wt.\% and 1 wt.\%) aqeous solution was used as outer phase and pure water was used as inner phase. Both liquids were introduced to the chip via inlets 1 and 3 respectively (see Figure 5). Two component Sylgard 184 silicone kit mixed in ratio 3:2 was used as middle phase and was injected via inlet 2. In the first step of the process the outer phase was introduced to the system followed by injection of middle phase. After the silicone droplet formation (at junction $A)$ was balanced, the inner phase liquid was slowly injected $\left(Q_{1}>Q_{2}>Q_{3}\right.$, where $Q$ refers to liquid flow rate) increasing the flow rate until the intended size of the microcapsule core was obtained. The resulting double emulsion was collected and was left for 24 hours at room temperature for the shell of the core-shell microspheres to be crosslinked and form a solid layer. Subsequently the double emulsion was heated up to $50{ }^{\circ} \mathrm{C}$ for 1 hour to ensure full crosslinking of PDMS. Subsequently, the collected core shell microspheres were transferred onto large petri dish and left for drying for 5 hours at room temperature. 


\subsection{Preparation of PDMS films with incorporated liquid core microspheres.}

Various amounts of dried liquid core microcapsules were mixed with different commercial PDMS compositions and carefully transferred onto a metal mold with $1 \mathrm{~mm}$ thick spacers. The material was finally cured at $40{ }^{\circ} \mathrm{C}$ for 5 hours and additionally left overnight at room temperature for possible postcuring to take place. The PDMS crosslinking process was kept at lowest possible temperatures in order to avoid evaporation of encapsulated water that due to relatively high vapor pressure tends to migrate through the thin silicone shell. The microcapsules were mixed in various weight ratios $(10,20,30)$ with Sylgard 184 silicone kit mixed in ratio 13:1 (base : crosslinker) (all formulations are listed in Table 1). Significant excess of vinyl groups in the PDMS composition allows covalent bonding between PDMS network and PDMS shell of microcapsules possessing excess of silicone hydrides.

Table 1: Sample names with different amounts of incorporated core-shell microspheres with corresponding amounts of encapsulated filler.

\begin{tabular}{ccc}
\hline Sample name & $\begin{array}{c}\text { Amount of microcapsules incorporated } \\
\text { into PDMS film [wt. \%] }\end{array}$ & $\begin{array}{c}\text { Amount of filler (water) incorporated } \\
\text { into PDMS film [wt. \%] }\end{array}$ \\
\hline S-13:1 & 0 & 0 \\
S-13:1_10 & 10 & 1.5 \\
S-13:1_20 & 20 & 3 \\
S-13:1_30 & 30 & 4.5 \\
\hline
\end{tabular}

\subsection{Characterization methods}

Leica DM LB optical microscope was used to determine diameter and morphology of single and double emulsions as well as PDMS films containing various amounts of microcapsules. All PDMS composites were studied in terms of mechanical and electrical properties. Frequency sweep tests performed on Ares G2 TA Instruments rheometer were conducted in order to determine both storage and loss moduli of investigated compositions. $25 \mathrm{~mm}$ of diameter and $1 \mathrm{~mm}$ thick disc samples were tested in the frequency range between $100 \mathrm{~Hz}$ and $0.001 \mathrm{~Hz}$ and under controlled strain mode (2\% strain) at room temperature. Novocontrol broadband dielectric spectrometer was used to investigate dielectric permittivity, dielectric losses and conductivity of samples. $20 \mathrm{~mm}$ of diameter and $1 \mathrm{~mm}$ thick disc specimens were tested in the frequency range between $10^{6} \mathrm{~Hz}$ and $10^{-1} \mathrm{~Hz}$.

\section{RESULTS AND DISCUSSION}

\subsection{Preparation of PDMS-microcapsules composites}

The produced core-shell microspheres used for preparation of composites had average diameter and core diameter of $350 \mu \mathrm{m}$ and $200 \mu \mathrm{m}$ respectively (Figure 7). These relatively large dimensions of microcapsules comparing to the designed film thickness result from technical limitations of microfluidic chip and viscosity of middle phase in double emulsion which in this case was off-stoichiometric mixture of Sylgard 184 silicone kit. It is believed that by decreasing dimensions of microfluidic chip channels and introducing liquids of lower viscosities smaller microcapsules could be obtained. 

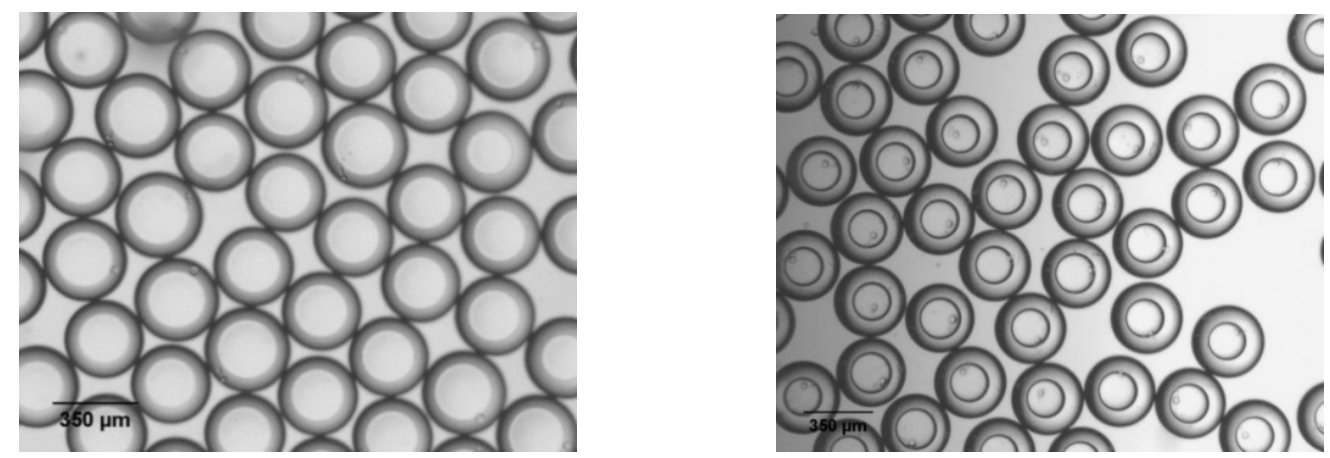

Figure 7: Microscope images of the microcapsules obtained from water in silicone in water double emulsions.

The liquid-core microcapsules were successfully incorporated within PDMS network forming stable films. Optical microscope images confirm that the microcapsules were well distributed and that water was still present in the capsules although the samples were exposed to elevated temperatures during PDMS crosslinking process. Black spots that can be seen in Figure 8 correspond to air bubbles which indicate that, to some extent, water evaporated from the core of the spheres. Nevertheless the fraction of empty capsules is acceptable and in case of samples with smaller amounts of filler becomes negligible. Specimens with higher microcapsules content are more influenced by emerging air voids since the percentage amount of filler in the polymer film becomes larger. Relatively high vapor pressure of water and its polar nature are the two main factors that are responsible for complications in handling of PDMS-water core microcapsules composites.
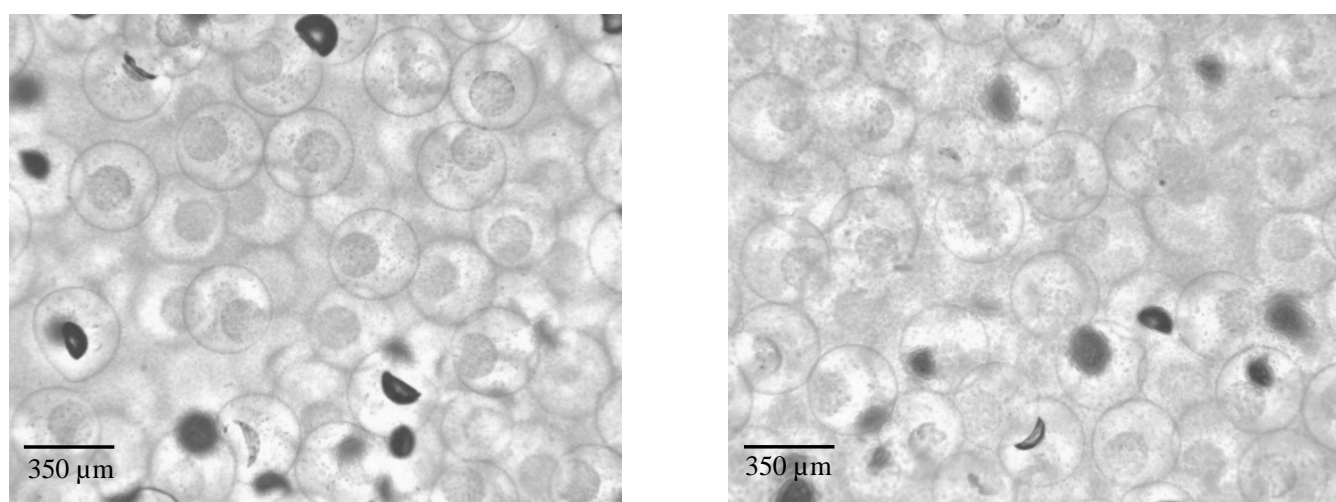

Figure 8: Microscope images of crosslinked PDMS films with incorporated core-shell microspheres. Dark spots present in the matrix are most likely air voids.

\subsection{Mechanical properties}

The prepared films are stable and free-standing at the current film thicknesses and have considerable strength when handling them. This implies that there is a significant crosslinking of the microcapsules into the elastomer as was also concluded from Gonzalez et al. [11]. Frequency sweep tests were conducted in order to determine viscoelastic properties of prepared composites and characterize how increasing amounts of core-shell microspheres influence mechanical properties. Rheology measurements were carried out on four different specimens and results summarizing changes of storage modulus and tan delta as function of frequency were plotted in Figure 9. 

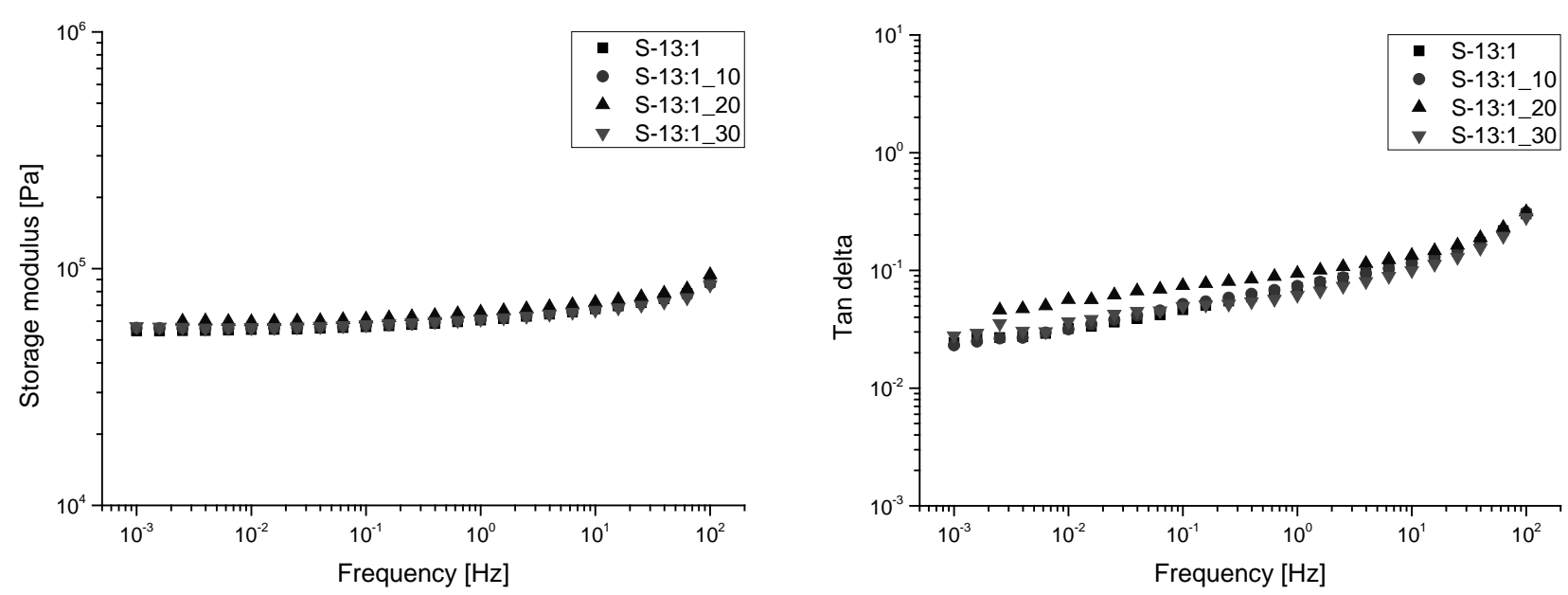

Figure 9: Storage modulus (left) and tan delta (right) of unfilled elastomer and different PDMS-microcapsules composites obtained from frequency sweep tests performed at controlled strain mode at $2 \%$ strain. The S-13:1 is the pure off-stoichiometric mixture of Sylgard 184.

It can be seen clearly that curves representing storage moduli of all tested compositions indicate nearly identical elastic behavior when applying stress. In all cases the elastic modulus at plateau region (from $10^{-1}-10^{-3} \mathrm{~Hz}$ ) varies from $55 \mathrm{kPa}$ to $60 \mathrm{kPa}$. These small differences are within the range of measurement error so it can be assumed that incorporation of core-shell microspheres of the structure described in our study does not influence significantly the viscoelastic behavior of tested PDMS composition. This observation is confirmed by tan delta of tested samples plotted as function of frequency, which, as can be seen in Figure 9, remains also nearly unchanged with increasing amounts of incorporated microcapsules.

When interpreting this phenomenon it has to be mentioned that there are few factors influencing viscoelastic properties of the discussed formulations. On one hand a substance of dominant viscous behavior and negligible elastic behavior is incorporated into PDMS network which in this case is water (and also to some extend air), and on the other hand we deal with more rigid structure of microcapsules shell which locally enhances elasticity of composites. These two coexisting and competitive factors determine the resulting overall viscoelastic behavior of tested composites.

\subsection{Dielectric spectroscopy measurements}

As described previously, the main reason for introducing microcapsules into PDMS films is to enhance the relative permittivity of the final product without compromising its other dielectric and mechanical properties. Results of investigating of this parameter are summarized in Figure 10. The dielectric constant of Sylgard 184 mixed in ratio 13:1 was successfully increased by incorporation of water-core microcapsules. The values measured at frequency of $10^{3} \mathrm{~Hz}$ indicate an increase from 2.8 for unfilled network to 3.6 for composite containing $30 \mathrm{wt}$ \% of microcapsules (4.5 wt.\% of water). These are very promising values in context of possible decrease of dielectric constant caused by the presence of air voids in the structure - higher amount of air bubbles for higher loading of microcapsules in the sample. By eliminating empty microcapsules the dielectric permittivity is expected to increase even more making PDMS-water composites competitive for other high permittivity materials. Dielectric spectroscopy measurements proved also that the dielectric loss of each specimen is at the same level in the function of frequency. 

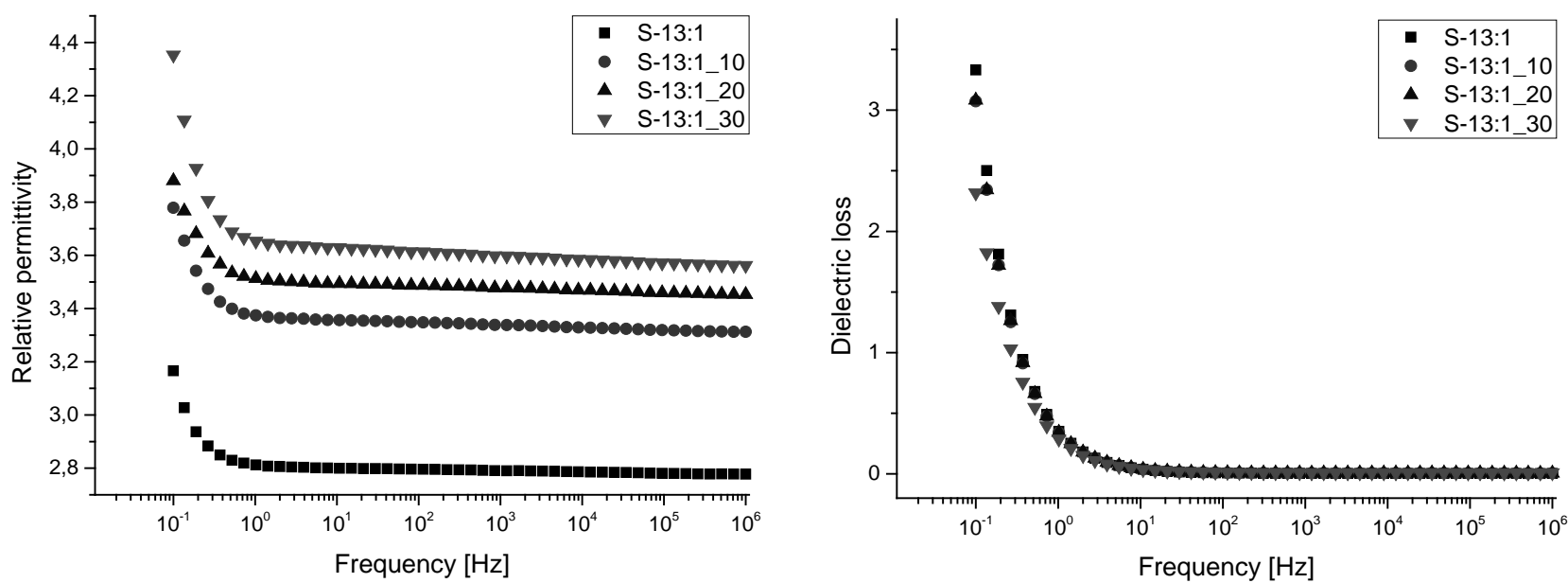

Figure 10: Relative permittivity and dielectric loss of elastomer reference and different PDMS-microcapsules composites as a function of frequency measured at room temperature.

Conductivity was proved to remain at nearly same level for all tested compositions (Figure 11). Although all samples exhibit similar conductivity, small deviations in the high frequency region can be observed. As expected the lower the filler loading the lower the conductivity. This phenomenon is in perfect agreement with presumptions which implied that water, as a high conductivity filler potentially decreasing resistivity of materials, does not influence significantly the conductivity which is explained by the fact that each portion of water in the investigated systems was hermetically encapsulated within PDMS insulating shell and therefore conductive pathways throughout the material were unlikely to appear and wherefore the overall conductivity remained almost unaffected.

At this step we decided to resign on investigating PDMS-microcapsules composites in terms of breakdown strength and their ultimate mechanical properties like tear strength and maximum elongation. It is believed that attempts of determination of these parameters would give unreliable outcome due to relatively high thickness of prepared samples.

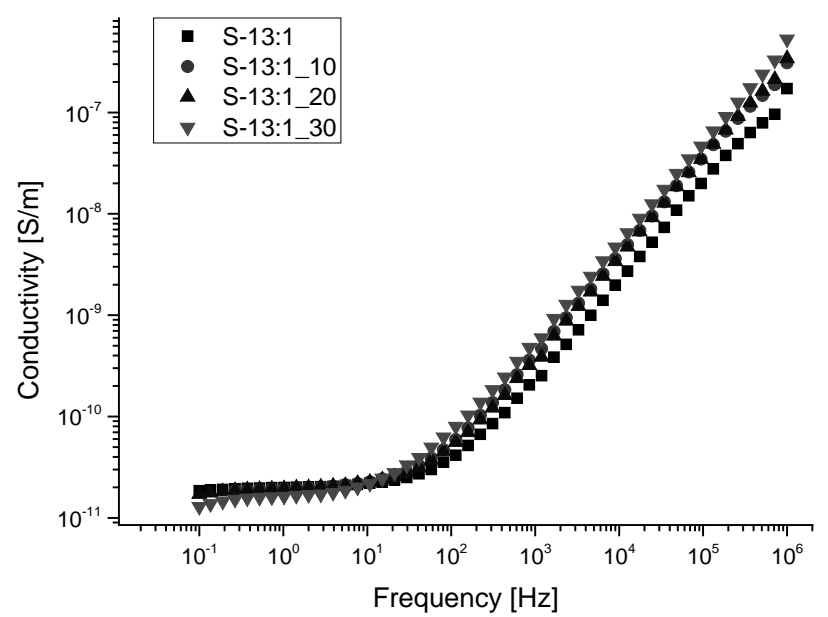

Figure 11: Conductivity of different PDMS-microcapsules composites as a function of frequency measured at room temperature. 


\section{CONCLUSIONS}

In this study we present an easy and efficient way of preparation of liquid-core microcapsules with PDMS forming solid insulating shell encapsulating high permittivity polar liquids. The thiol-ene flow-focusing microfluidic system was proved to be a powerful tool capable of producing microspheres of extremely narrow size distribution and various sizes of individual layers. Encapsulated water was successfully incorporated into the PDMS matrix increasing its relative permittivity without compromising dielectric losses and resistivity. Mechanical properties remained also unaffected by different microcapsule loadings in the investigated composites.

Although these pioneering attempts of incorporation of polar liquids into silicone networks gave very promising results there are many parameters and steps in the preparation procedure that require further optimization. We believe that through incorporation of smaller microcapsules with thinner shells and by eliminating of hollow spheres the overall performance of this type of composites can be further enhanced.

\section{ACKNOWLEDGEMENTS}

We acknowledge financial support from DTU Proof of Concept as well as from the Danish Advanced Technology Foundation.

\section{REFERENCES}

[1] Goswami, K., Galantini, F., Mazurek, P., Daugaard, A. E., Gallone, G., Skov, A. L., "Reinforced poly(propylene oxide): a very soft and extensible dielectric electroactive polymer”, Smart Mater. Struct. 22, 115011 (2013)

[2] Skov, A. L., Vuduagiri, S., Benslimane, M. Y., "Novel silicone elastomer formulations for DEAPs”, Proc. of SPIE, 8687, 86871I-1 (2013)

[3] Galantini, F., Bianchi, S., Castelvetro, V., et al., "Functionalized carbon nanotubes as a filler for dielectric elastomer composites with improved actuation performance”, Smart Mater. and Struct., 22, 5, 055025 (2013)

[4] Racles, C., Cazacu, M., Fischer, B., Opris, D. M., "Synthesis and characterization of silicones containing cyanopropyl groups and their use in dielectric elastomer actuators”, Smart Mater. Struct. 22, 104004 (2013)

[5] Madsen, F. B., Daugaard, A. E., Hvilsted, S., Skov, A. L., "Dipolar cross-linkers for PDMS networks with enhanced dielectric permittivity and low dielectric loss”, Smart Mater. Struct. 22104002 (2013)

[6] Madsen, F. B., Dimitrov, I., Daugaard, A. E., Hvilsted, S., Skov, A. L., "Novel cross-linkers for PDMS networks for controlled and well distributed grafting of functionalities by click chemistry”, Polymer Chem., 4 (5), 1700 1707 (2013)

[7] Molberg, M., Crespy, D., Rupper, P., Nüesch, F., Månson, J. A. E., Löwe, C., Opris, D. M., "High Breakdown Field Dielectric Elastomer Actuators Using Encapsulated Polyaniline as High Dielectric Constant Filler”, Adv. Funct. Mater., 3280-3291 (2010)

[8] Larsen, A. L., Hansen, K., Sommer-Larsen, P., Hassager, O., Bach, A., Ndoni, S., Jørgensen, M., “Elastic Properties of Nonstoichiometric Reacted PDMSNetworks”, Macromolecules, 36, 10063 (2003)

[9] Abate, A. R., Weitz, D. A., "Faster multiple emulsification with drop splitting”, Lab Chip, 11, 1911 (2011)

[10] Carlborg, C. F., Haraldsson, T., Öberg, K., Malkoch, M., van der Wijngaart, W., "Beyond PDMS: off-stiochiometry thiol-ene (OSTE) based soft lithography for rapid prototyping of microfluidic devices”, Lab Chip, 11, 3136 (2011)

[11] Gonzalez, L., Kostrzewska, M., Ma, B., Hansen, H., Li, L., Hvilsted, S., Skov, A. L., "Preparation and Characterization of Thermoplastic Encapsulated PDMS Microspheres with Reactive Handles”, Macromol. Mater. and Engin. (2013) 